\title{
Design of an omnidirectional single-point photodetector for large-scale spatial coordinate measurement
}

\author{
Hongbo Xie ${ }^{a}$ and Chensheng $\mathrm{Mao}^{a}$ and Yongjie Ren ${ }^{b}$ and Jigui $\mathrm{Zhu}^{b}$ and Chao \\ Wang $^{c}$ and Lei Yang ${ }^{a \dagger}$ \\ ${ }^{a}$ College of Precision Instrument and Opto-electronics Engineering, Tianjin \\ University
}

Key Laboratory of Optoelectronics Information Technology, Ministry of Education, Tianjin, 300072, China;

${ }^{b}$ State Key Laboratory of Precision Measuring Technology and Instruments, Tianjin University, Tianjin 300072, China;

${ }^{c}$ School of Engineering and Digital Arts, University of Kent, Canterbury CT2 7NT, UK

\begin{abstract}
In high precision and large-scale coordinate measurement, one commonly used approach to determine the coordinate of a target point is utilizing the spatial trigonometric relationships between multiple laser transmitter stations and the target point. A light receiving device at target point is the key element in large-scale coordinate measurement systems. To ensure high-resolution and highly-sensitive spatial coordinate measurement, a high-performance and miniaturized omnidirectional single-point

${ }^{\dagger}$ E-mail: yanglei@tju.edu.cn
\end{abstract}


photodetector (OSPD) is greatly desired. Here we report one design of OSPD using aspheric lens, which achieves enhanced reception angle of $-5^{\circ}$ to $45^{\circ}$ in vertical and $360^{\circ}$ in horizontal. As the heart of our OSPD, the aspheric lens is designed in geometric model and optimized by LightTools Software, which enables to reflect wide-angle incident light beam into the single-point photodiode. The performance of home-made OSPD is characterized with working distances from $1 \mathrm{~m}$ to $13 \mathrm{~m}$ and further analyzed utilizing developed geometric model. The experimental and analytic results verify that our new device is highly suitable for large-scale coordinate metrology. The developed device also holds great potential in various applications such as omnidirectional vision sensor, indoor global positioning system and optical wireless communication systems.

Keywords: Omnidirectional single-point photodetector, aspheric lens, large-scale coordinate metrology

\section{INTRODUCTION}

Three-dimensional coordinate metrology within large-scale volume is a popular and extensive topic today, which offers great support to equipment manufacturing industry and precision engineering. ${ }^{1,2}$ With rapidly developing of pillar industry, such as aviation, shipbuilding, iron and transportation, the demand for higher performance 
large-scale spatial coordinate measuring technology has become paramount. Measuring with multi-station and multi-point, automatically identifying target points and building global network are the central and existing techniques for large-scale coordinate metrology, which are always based upon the principle of laser scanning trigonometric measurement system and especially useful for calculating spatial locations. ${ }^{3,4}$

In normal laser scanning trigonometric measurement arrangement (see Fig. 1), multiple transmitter stations are employed to emit the laser planes with $360^{\circ}$ scanning angle in horizontal and wide angle in vertical. The three-dimension coordinate of scanned target point is normally converted by scanning angles and positions of transmitter stations. ${ }^{4-6}$ A scanning angle of target point relative to one transmitting station is obtained by recording the exact time when laser plane rotates from fixed original position to the target point. ${ }^{6}$ In detail, this transmitting station emits another pulsed light as the synchronous signal when it starts scanning, and the laser plane received by photodetector is regarded as the stop signal. The scanning angle can be calculated by the time difference between start and stop signal and rotating speed. Therefore, small-sized omnidirectional detector, being placed at the location of target point, is extremely desirable for measurement system, which has the avail- 
ability of receiving laser beams with $360^{\circ}$ in horizontal and wide angle in vertical from multi-station simultaneously.

There are several approaches to build a compact omnidirectional detector. Apparently, the most straightforward way is to make the active area of the photodiode into a spherical shape, which can provide $360^{\circ}$ field of view (FOV) both in horizontal and vertical plane. Unfortunately, a spherical photodiode suffers from low working speed (normally less than $1 \mathrm{MHz}$ ) and low sensitivity $(-36.45 \mathrm{dBm})$ due to the enlarged active area and massive dark current. ${ }^{7-10}$ The reported small-sized spherical photodiode with $100 \mathrm{~mm}$ diameter is not beneficial for enhancing the positioning accuracy in spatial metrology ${ }^{7}$ which should be further reduced the volume but will bring difficulties in manufacture. On the other hand, the fisheye lens having short focus and wide field of view $\left(90^{\circ} \sim 180^{\circ}\right)$ is an alternative approach to design an omnidirectional detector with normal flat photodiode. ${ }^{11,12}$ Distinct advantages for fisheye photodiode include fast detection (several MHz) and high sensitivity $(\sim-50$ $\mathrm{dBm}) .{ }^{11,13}$ However, severe optical field distortion and complex structure limit its application in precision positioning. ${ }^{11-14}$ As a consequence, highly efficient light collection design is a real demand to facilitate the widespread utilities of large-scale coordinate measurement. 


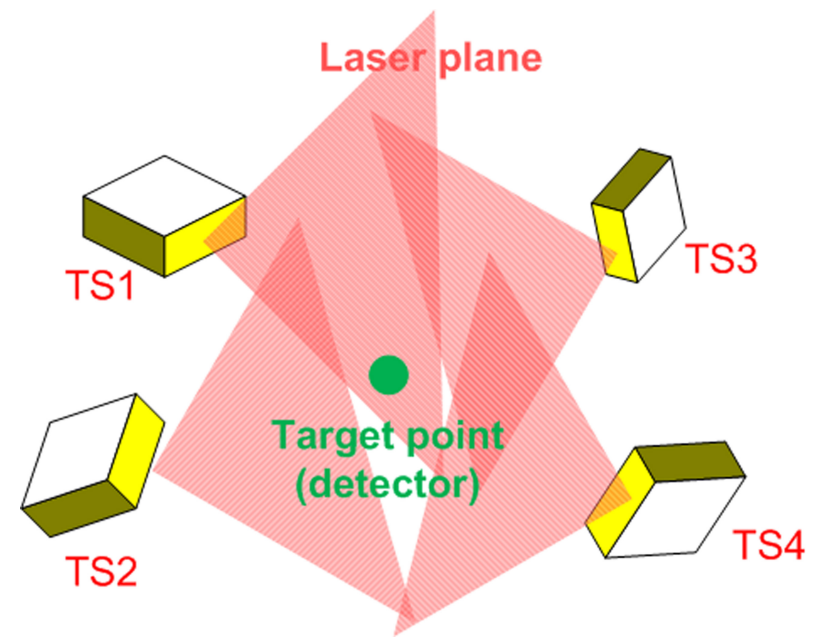

Figure 1. Principle of large-scale spatial coordinate measurement based on opto-electronic scanning. TS, transmitter station.

Panoramic camera, ${ }^{15,16}$ mainly consisting of a classical camera and a convex mirror, covers a large field of view and sees in all directions, which brings some enlightenment to address this challenge. In this paper, we report a design of OSPD using specially designed and optimized aspheric lens. We experimentally demonstrate successful detection of vertical $-5^{\circ}$ to $45^{\circ}$ and horizontal $360^{\circ}$ incident laser beams. The work speed and detection sensitivity of OSPD could achieve $25 \mathrm{MHz}$ and $-58 \mathrm{dBm}$, respectively. The package size of $49 \mathrm{~mm}$ diameter and $95 \mathrm{~mm}$ height and photodiode with $8.26 \mathrm{~mm}$ diameter and $4.07 \mathrm{~mm}$ height satisfy the requirement of large-scale spatial coordinate measurement system. The remainder of this paper is structured as follows. The 2D geometric model of OSPD and its operation principle of focusing incident light with wide angle into a single-point detector are described particularly in 
Sec. II. Optimization of aspheric surface design is also investigated using LightTools Software. The utility of designed OSPD is verified in Sec. III, where the machined OSPD is presented and its performance in wide-angle light detection with different working distances is tested. A detailed theoretical analysis that predicts the results of OSPD has been developed and its accordance with experimental data illustrates the feasibility of proposed method. Finally, we summarize our findings and discuss implications and future directions for this work.

\section{PRINCIPLE}

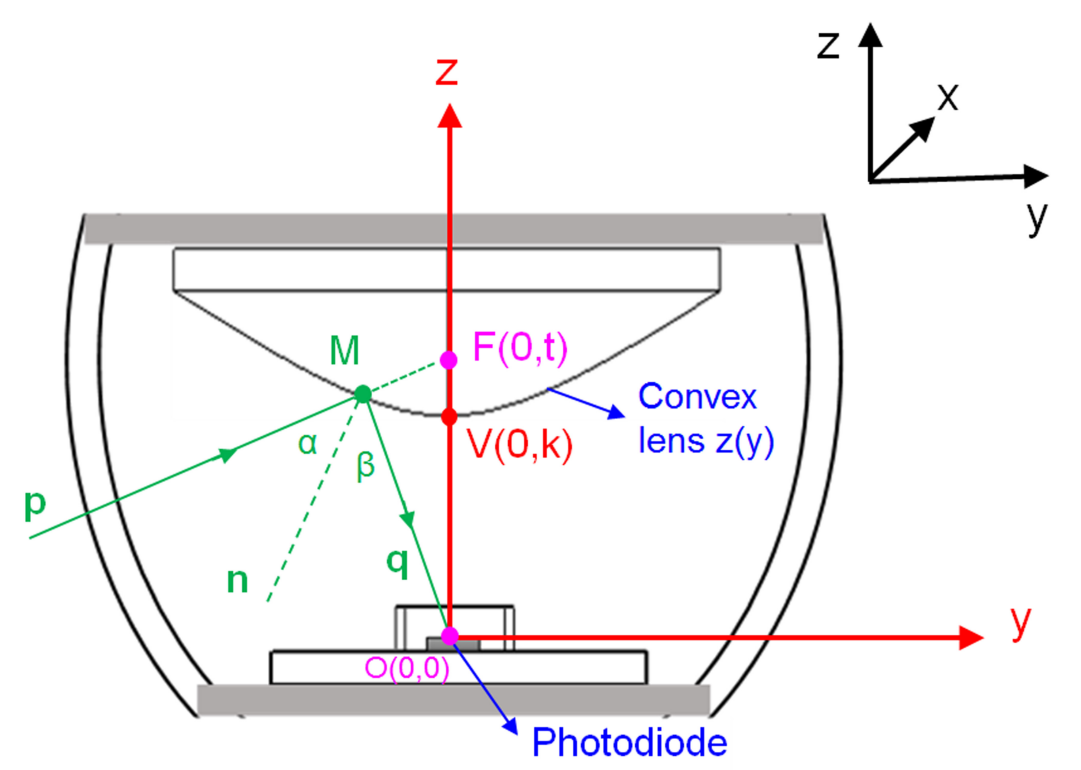

Figure 2. Geometric model of OSPD, composed of a convex lens and a photodiode, in the coordinate system. Point $\mathrm{O}, \mathrm{V}, \mathrm{F}$ denote the origin, vertex and focus point, respectively. $\mathbf{p}, \mathbf{q}, \mathbf{n}$ are the unit vectors of incident ray, reflected ray and normal of the surface.

Sec. 2 mainly presents the fundamental principle to design the OSPD in geometric 
model $^{15,16}$ and specially optimize this device in LightTools Software. Assuming the scanning laser beam is a ideal plane in $\mathrm{Y}-\mathrm{Z}$ coordinate system, the $2 \mathrm{D}$ geometrical model of the OSPD is established in $Y Z$ plane, which consists of an unknown surface with rotationally symmetrical structure and a photodiode mounted on the receiving plane $(Z=0)$ (see Fig. 2). The incident ray, which passes the focus point $\mathrm{F}$ of lens, is reflected by the surface and then goes through the true image point O. The photodiode is located at the image point, which is defined as the origin of $Y-Z$ coordinate system $(0,0)$. The incident point on surface $M$, focus point $F$ and vertex $\mathrm{V}$ are represented by $(y, z(y)),(0, t)$ and $(0, k)$, respectively, where $z(y)$ is the function of unknown surface. The incident angle and reflected angle of the scanning ray are defined as $\alpha$ and $\beta$, respectively. Moreover, $\mathbf{n}$ is the normal of surface and $z^{\prime}(y)$ is the slope of tangent line passing point M.

The unit vector of incident ray $\mathbf{p}$ and reflected ray $\mathbf{q}$ can be respectively written as

$$
\begin{gathered}
\mathbf{p}=\left[\frac{y}{\sqrt{y^{2}+(t-z(y))^{2}}}, \frac{t-z(y)}{\sqrt{y^{2}+(t-z(y))^{2}}}\right] \\
\mathbf{q}=\left[\frac{y}{\sqrt{y^{2}+z(y)^{2}}}, \frac{z(y)}{y^{2}+z(y)^{2}}\right]
\end{gathered}
$$


Meanwhile, the unit normal vector $\mathbf{n}$ of surface takes the form

$$
\mathbf{n}=\left[\frac{z^{\prime}(y)}{\sqrt{1+z^{\prime}(y)^{2}}},-\frac{1}{\sqrt{1+z^{\prime}(y)^{2}}}\right]
$$

According to the vector theory, the incident angle $\alpha$ and reflected angle $\beta$ satisfy $\cos \alpha=\mathbf{p} \cdot \mathbf{n}$ and $\cos \beta=\mathbf{q} \cdot \mathbf{n}$, respectively. In Snell's Law, $\sin \alpha=\sin \beta$, therefore

$$
\cos ^{2} \alpha=\cos ^{2} \beta
$$

Substituting Eq. (1)-(3), Eq. (4) becomes

$$
\left(\frac{y z^{\prime}(y)+z(y)-t}{\sqrt{\left(y^{2}+(t-z(y))^{2}\right)\left(1+z^{\prime}(y)^{2}\right)}}\right)^{2}=\left(\frac{y z^{\prime}(y)-z(y)}{\sqrt{\left(y^{2}+z(y)^{2}\right)\left(1+z^{\prime}(y)^{2}\right)}}\right)^{2}
$$

After simplifying

$y^{2}(y-t)^{2} z^{\prime}(y)^{2}-2\left(2 y z(y)^{3}-3 t y z(y)^{2}+\left(2 y^{3}+t^{2} y\right) z(y)+y^{3}\right) z^{\prime}(y)+2 t z(y)-t^{2} y^{2}=0$

which is a first-order nonlinear differential equation with boundary condition $z(0)=$ 
$k$ and $z^{\prime}(0)=0$. One particular solution of Eq. (6) is found to be

$$
z(y)=\frac{1}{2}(t-2 k)\left(1-\sqrt{1-\frac{y^{2}}{k(k-t)}}\right)
$$

Multiplying $\frac{1+\sqrt{1-\frac{y^{2}}{k(k-t)}}}{1+\sqrt{1-\frac{y^{2}}{k(k-t)}}}$ by the first item of Eq. (7), the function $z(y)$ can also be expressed in the standard form of quadric surface, which is given by

$$
z(y)=\frac{C y^{2}}{1+\sqrt{1-(1+K) C^{2} y^{2}}}
$$

where $K=-\left(\frac{t}{t-2 k}\right)^{2}$ is the conic constant and $C=\frac{2 k-t}{2 k(t-k)}$ is the curvature of the surface.

Based on theoretical model above, the model of OSPD with a convex lens having quadric surface is established in the Software of LightTools 7.1, as shown in Fig. 3 (a). Taking into account the demand of laser plane scanning in large-scale measurement system, an OSPD is required to response the light beam with vertical $-5^{\circ}$ to $45^{\circ}$ view. The light spot, known as the true image point, on the photodiode is particularly investigated when the the incident angle $\theta$ ranges from $-5^{\circ}$ to $45^{\circ}$. With the purpose of eliminating the effect induced by the shell of OSPD, which slightly changes the optical path of incident light, high-order items are also introduced into the function 
of $z(y)$. Guaranteeing all the light spots simultaneously reaching the minimum sizes through adjusting the constants of $K, C$ and high-order coefficients (see Fig. $3(\mathrm{~b})$ ), the function of optimized surface can be expressed as $z(y)=\frac{y^{2}}{10.108\left(1+\sqrt{1-(1-5.0034) y^{2} / 10.108^{2}}\right)}+1.8885 \times 10^{-6} y^{4}+4.7079 \times 10^{-9} y^{6}-9.956 \times 10^{-12} y^{8}$

As a consequence, the assumed unknown surface is identified as an aspheric surface with rotationally symmetric structure. According to $C=1 / 10.108 \mathrm{~mm}$ and $K=$ -5.0034 , the key parameters defined in geometric model of OSPD are $k=8.1725 \mathrm{~mm}$ and $t=11.2953 \mathrm{~mm}$.
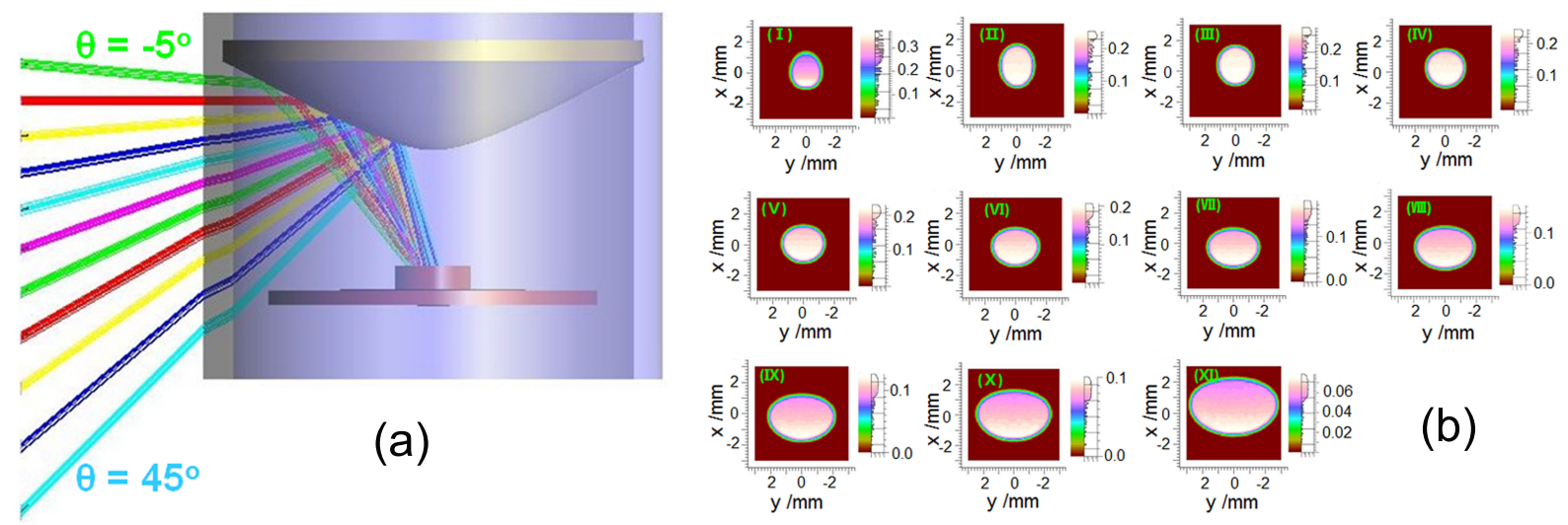

Figure 3. Model of OSPD established in LightTools Software and its simulation results. (a) The ray-tracing model of OSPD when the angle of incident light $\theta$ ranges from $-5^{\circ}$ to $45^{\circ}$. The incident angle $\theta$ difference between adjacent light ray is $5^{\circ}$. (b) Spot pattern of light incoming the photodiode. The graphs (I) to (XI) originates from light with $-5^{\circ}$ to $45^{\circ}$ incident angle, when the difference of $\theta$ between two adjacent graphs is $5^{\circ}$. The legend representing the irradiation illumination of incident light with unit of $\mathrm{W} / \mathrm{mm}^{2}$. 


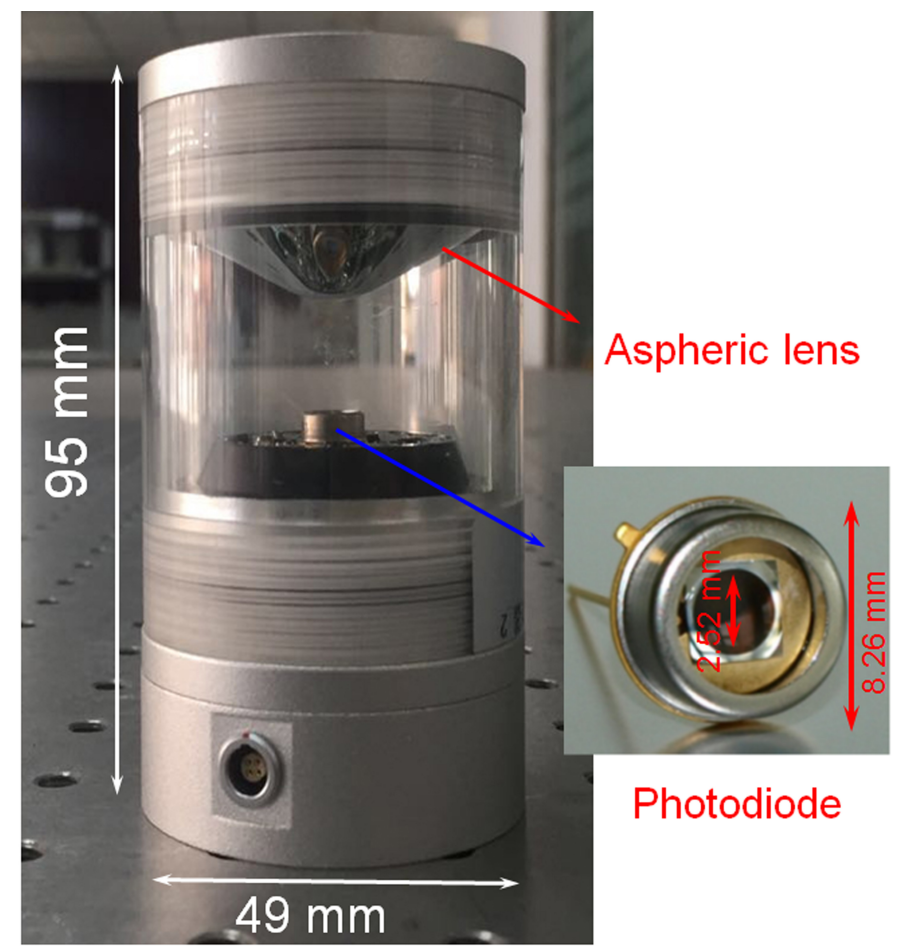

Figure 4. A photograph of assembled OSPD marked by outline dimension, consisting of a coated optical aspheric lens and a high-performance photodiode (First Sensor, PC5-6-TO5). The diameter of active area on photodiode is $2.52 \mathrm{~mm}$.

\section{EXPERIMENTS AND DISCUSSIONS}

The photograph of assembled OSPD, consisting of a coated aspheric lens and a photodiode, is shown in Fig. 4. The machined optical aspheric lens, with shape function of Eq. (9), has rotationally symmetric structure and diameter $S$ is $38 \mathrm{~mm}$. The commercial photodiode (First sensor, PC5-6-TO5) with $5 \mathrm{~mm}^{2}$ active area $(D=2.52$ $\mathrm{mm}$ ) and $900 \mathrm{~nm}$ peak response wavelength is mounted on the receiving plane while the OSPD is packaged in the glass shell. The distance between aspheric lens and photodiode is chosen at $26 \mathrm{~mm}$, which is the observed highest efficiency position for 
photodiode.

In order to characterize the home-made OSPD, experimental arrangement composed of transmitter station (TS), oscilloscope (OSP) and amplifier (AMP) is illustrated schematically in Fig. 5(a). Stable optical plane beam, outputting from TS, is generated by mounting a pulsed laser device (OSRAM, SPLLL90) on tum-table operated by $360^{\circ}$ rotating electric machinery. Plane-shaped pulsed laser beams with nearly $180^{\circ}$ emission angle in vertical, which is centered at $905 \mathrm{~nm}$ center wavelength and has $20 \mathrm{~nm}$ bandwidth, are received by new-designed OSPD. In addition, varying the relative angle $\theta$ between OSPD and TS, also standing for the vertical incident angle of laser beam, is realized by operating the height $H$ of OSPD using adjustable bracket. Before inputting into oscilloscope, the peak photocurrent $I_{d}$ exporting from OSPD is amplified by $20 \mathrm{~dB}$ when transimpedance amplifier OPA657 and operational amplifier LMH6624 integrated in AMP are used. A photography of experimental setup is depicted in Fig. 5(b).

Fig. 6 detailly presents the results of testing OSPD, which is characterized by amplified photocurrents $I\left(I=10^{2} I_{d}\right)$ from photodiode when the experimental parameters are varied. The power distribution of laser plane $P_{a}$, defined as power in unit interval ( $\mathrm{mw} / \mathrm{mm}$ ), is also measured (see the inset of Fig. 6), which is tied closed 

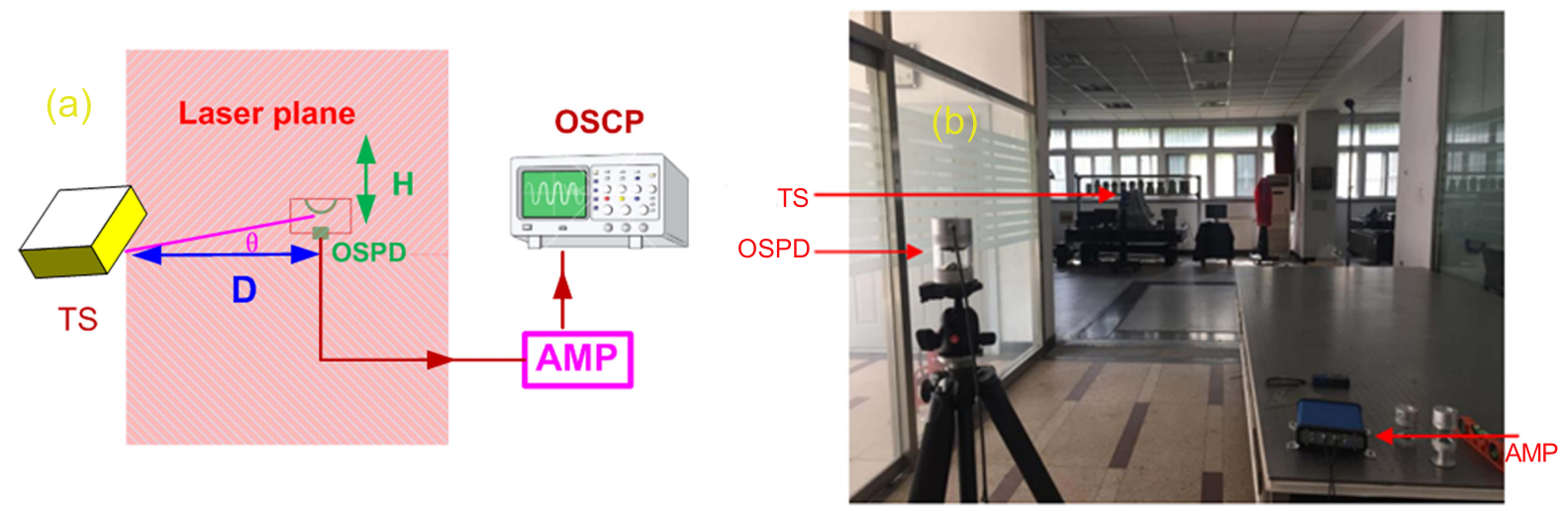

Figure 5. (a) Block diagram for testing the performance of OSPD. The signals outputting from OSPD are captured by oscilloscope (Tektronix, TDS2012B), when the relative angle $\theta$ between TS and OSPD (incident angle of laser beam) is varied by changing the relative working distance $D$ and height $H$. (b) A photograph of experimental setup in the lab. TS, transmitter station; Amp, amplifier; OSCP, Oscilloscope.

with distance $D$ and insensitive to incident angle $\theta$. Obviously, at same working distance, the photocurrents of OSPD with vertical angle $\theta$ from $-5^{\circ}$ to $45^{\circ}$ maintain high efficiency and almost keep consistency, otherwise, the photocurrents seriously drop down over the designed range. We also find out that the properties of OSPD in horizontal direction almost stay samely when it is revolved round the transmitter station in $360^{\circ}$. Meanwhile, general applicable scope of OSPD in our metrology system is ranging from $1 \mathrm{~m}$ to $10 \mathrm{~m}$. When the working distance $D$ between TS and OSPD exceeds $10 \mathrm{~m}$, the reason for reduction of photoncurrent is probably that power density $P_{a}$ severely decreases in the space. The above experiment verifies the feasibility of our OSPD to be used in large-scale coordinate metrology system. In addition, the glass shell slightly refracting the input light is non-ignorable, although 
the affection of shell having been considered in simulation and optimization (see.

Fig. 3(a)). Hollow shell for OSPD would be a better choice in future work.

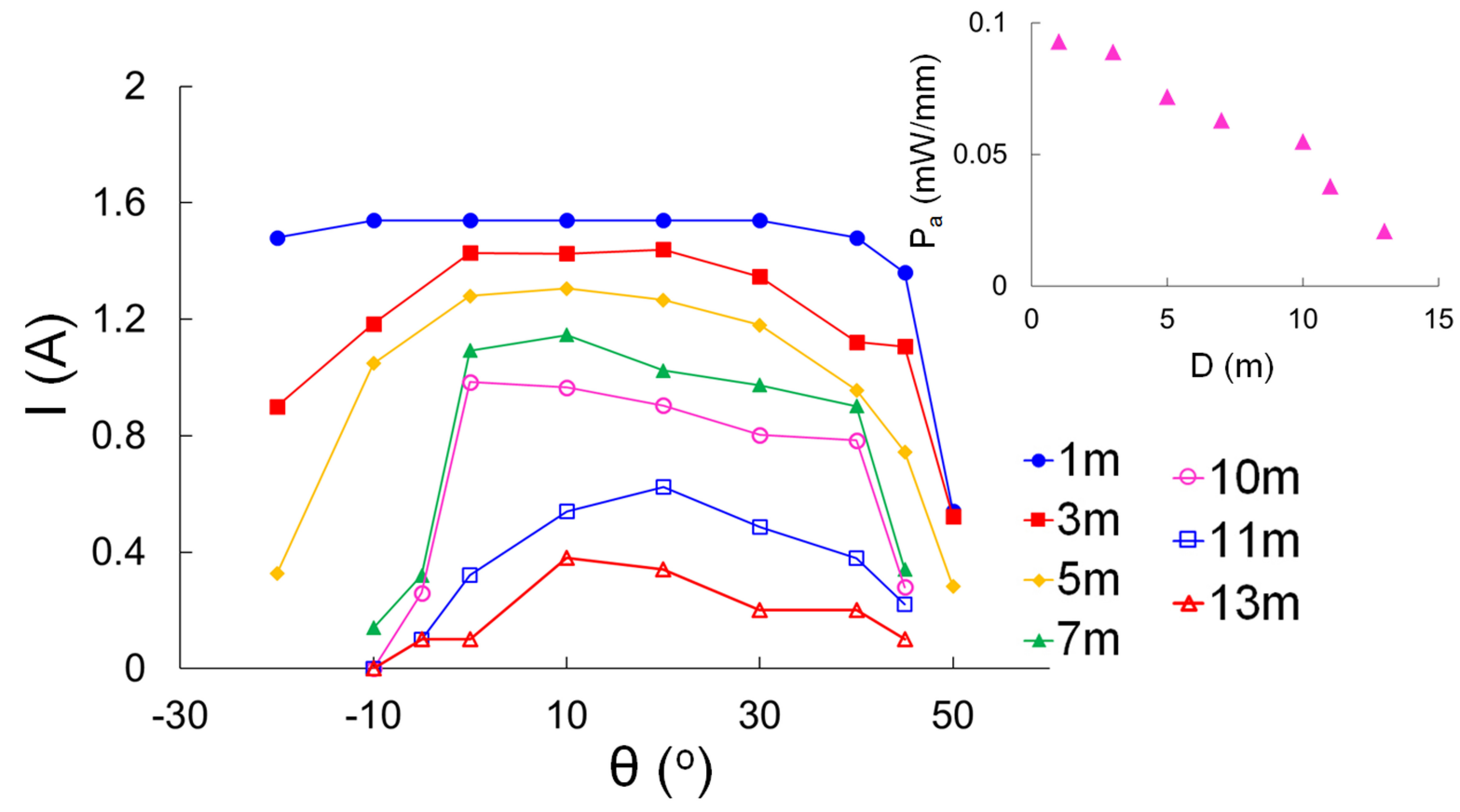

Figure 6 . The peak photocurrent $I$ outputting from OSPD versus the vertical incident angle $\theta$ of transmitter station when the relative working distance $D$ varies. Two-stage amplification circuit is employed for amplifying photocurrent by $20 \mathrm{~dB}$ before recording. The blue solid circle, red solid square, orange solid diamond, green solid triangle, purple hollow circle, blue hollow square, red hollow triangle represent the $D$ are $1 \mathrm{~m}, 3 \mathrm{~m}, 5 \mathrm{~m}, 7 \mathrm{~m}, 10 \mathrm{~m}, 11 \mathrm{~m}$ and $13 \mathrm{~m}$, respectively. The inset shows the measured average power of laser plane in unit interval $P_{a}$ when the distance $D$ between laser power meter (Ophir, Nova II) and transmitter station varies.

For clarity, the analysis on experimental arrangement will be presented in the following part. The geometrical model of experimental arrangement for testing OSPD (Fig. 5(a)) is built up in Fig. 7(a). It is reasonable to assume that all the incident rays are parallel to each other owing to the OSPD being approximately regarded as 

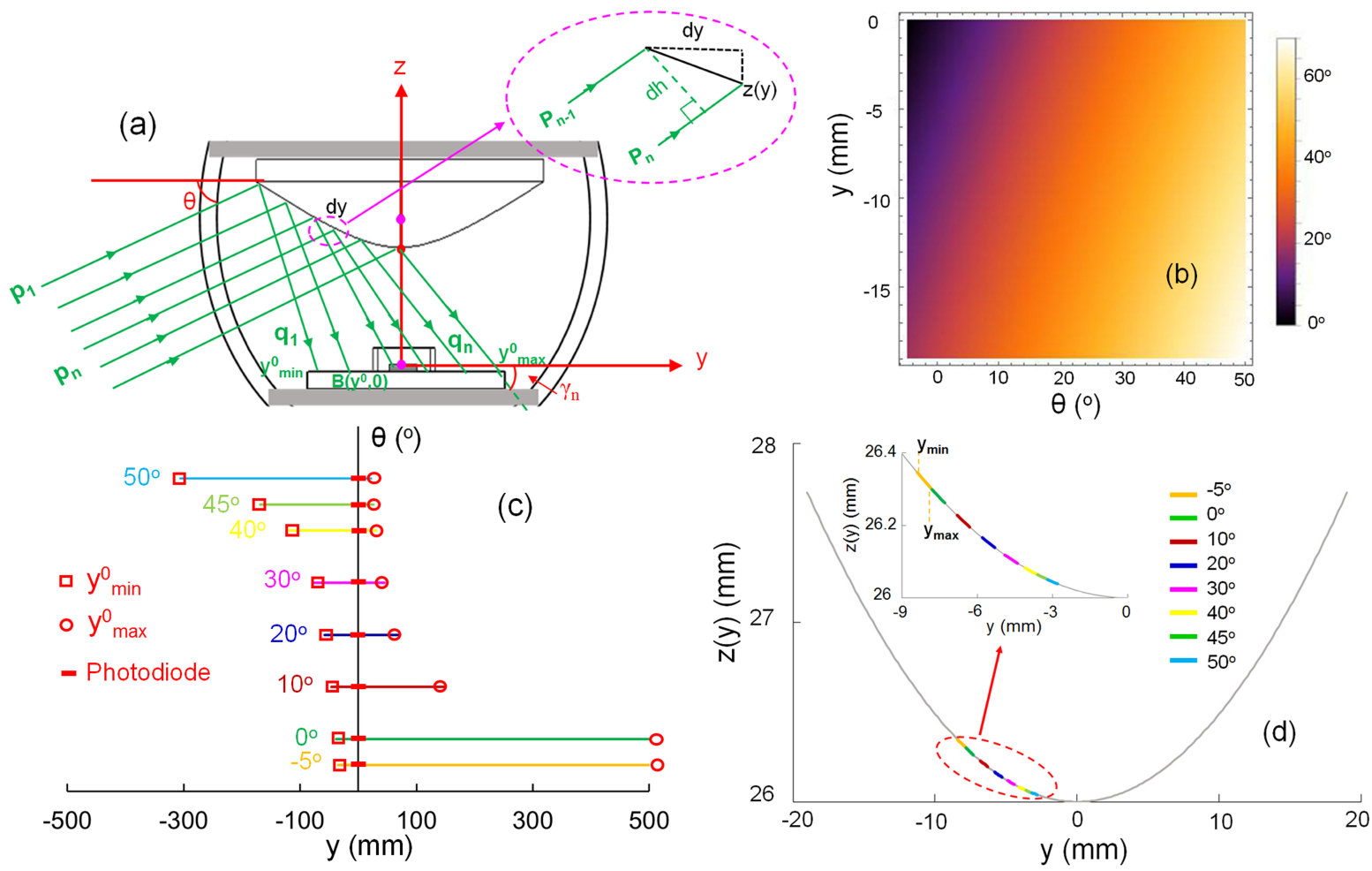

Figure 7. Detailed analysis on the experimental arrangement. (a) Geometrical model of OSPD with parallel rays incoming. (b) The density map of $\gamma_{n}$ versus the incident angle $\theta$ and coordinate $y$. (c) The distribution of light spot on the $Z=0$ versus the incident angle of OSPD $\theta$. The red bar denotes active area of photodiode on $Z=0$, which is from $-1.26 \mathrm{~mm}$ to $1.26 \mathrm{~mm}$. The red hollow square and circle stand for the maximum and minimum coordinate of light spot on $Z=0$, respectively. (d) The effective area of incident light on the aspheric surface, which accurately corresponds to the light spot in the active are of photodiode. Different color lines denote different incident angles, which are shown in the legend in detail. 
a point in laser plane. The unit vector of incident rays $\mathbf{p}_{n}(n=1,2,3 \ldots)$ has the form

$$
\mathbf{p}_{n}=[\sin \theta, \cos \theta]
$$

The unit vector of reflected rays $\mathbf{q}_{n}(n=1,2,3 \ldots)$ can be represented by

$$
\mathbf{q}_{n}=\left[\sin \left(-\gamma_{n}\right), \cos \left(-\gamma_{n}\right)\right]
$$

where $\gamma_{n}=\theta-2 \arctan \left(z^{\prime}(y)\right)$ is the included angle of reflected ray and $Z=0$ line. In addition, $z^{\prime}(y)$, standing for the first-order derivative of $z(y)$, is deduced to be

$$
z^{\prime}(y)=\frac{C y}{\sqrt{1-(1+K)(C y)^{2}}}
$$

when the high-order items are omitted. Eq. (12) represents the slope of tangent line passing point $\mathrm{M}(y, z(y))$. Moreover, $z^{\prime}(y)$ belongs to the range $(-1.75938,0)$ when $y \in(-19 \mathrm{~mm}, 0)$. The density map of $\gamma_{n}(\theta, y)$ with parameters angle $\theta$ and coordinate $y$ is plotted in Fig. 7(b), which clearly depicts the propagating directions of reflected rays.

Hence the incident point of laser beam $B\left(y^{0}, 0\right)$ in receiving plane, also considered 
as the intersection of incident ray and $Z=0$ line, can be expressed as

$$
\left(y+\frac{z(y)}{\tan \left(\gamma_{n}\right)}, 0\right)
$$

Therefore, the exact values of $y^{0}$, including the maximum value $y_{\max }^{0}$ and minimum value $y_{\min }^{0}$, can be calculated when the aspheric surface range in Y-axis is $y \in(-$ $19 \mathrm{~mm}, 0)$, which clearly show the scope of light spot in receiving plane $(Z=0)$. The light spots with range $\left(y_{\min }^{0}, y_{\max }^{0}\right)$ in different incident angle $\theta$ are shown in Fig. 7(c). In order to compare the size of light spot and active area of photodiode, the project of photodiode in the $Z=0$ direction with range $(-1.26 \mathrm{~mm}, 1.26 \mathrm{~mm})$ is also plotted using red bar. It is quite obvious that only the reflected rays falling into the active area of photodiode can be effectively converted to photocurrent. In view of the active range of photodiode and geometrical relationship between $\mathrm{M}(y, z(y))$ and $B\left(y^{0}, 0\right)$ (Eq. (13)), the effective incident regions $\left(y_{\min }, y_{\max }\right)$ on the aspheric surface are also calculated and plotted in Fig. 7(d).

According to the measured average power density of laser plane $P_{a}$, the theoretical value of peak photocurrent $I_{d}$ can be calculated based on the design model and analytic results shown in Fig. 7. Apparently, $d h$ is a significant parameter to calculate the total power of incident beam on the surface in the tiny range $d y$ (shown in the 

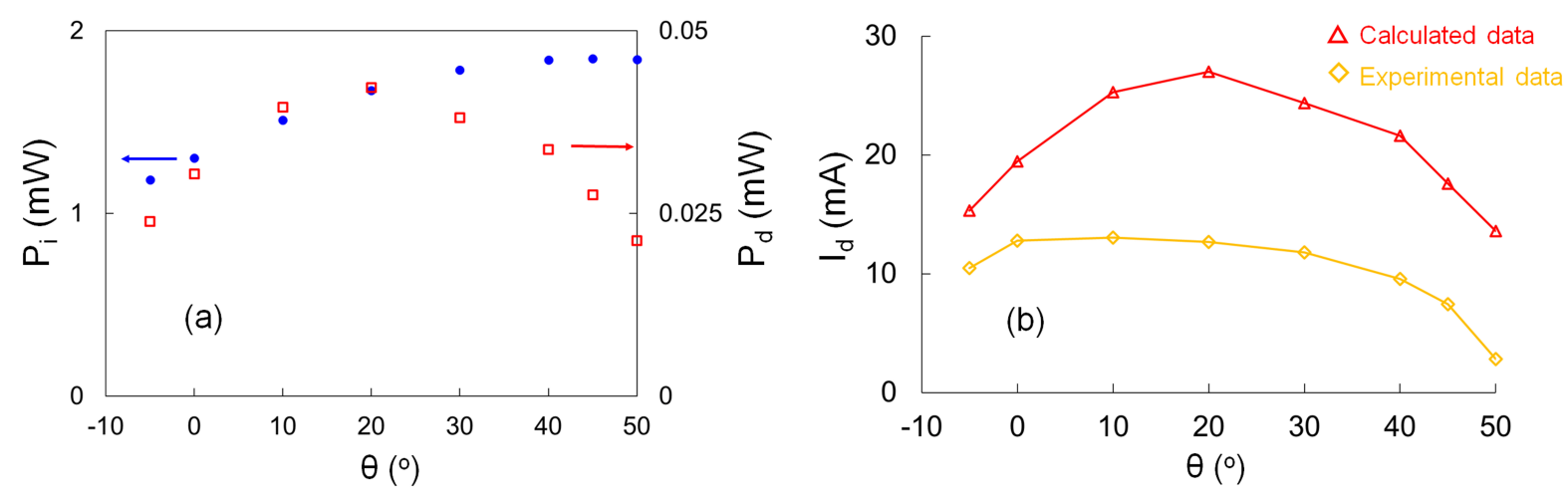

Figure 8. The numerical simulation results when the distance $D$ is fixed at $5 \mathrm{~m}$. (a) The average power of laser beam versus the incident angle $\theta$. The blue solid circle represents the total power of incident light collected by the entire aspheric surface $P_{i}$, corresponding to the primary axis. The red hollow square represents the power of reflected ray collected by photodiode $P_{d}$, corresponding to the secondary axis. (b) Photocurrent $I_{d}$ outputting from photodiode versus the incident angle $\theta$. The red hollow triangle represents the calculated photocurrent using the light power $P_{i}$ and experimental parameters. The yellow hollow diamond represents the obtained experimental data from Fig. 6 (yellow solid diamond) and relationship $I_{d}=10^{-2} I$.

marked part by pink dotted circle of Fig. 7(a)), which can be represented by

$$
d h=\frac{1}{\cos \arctan \left(z^{\prime}(y)\right)} \sin \left(\theta-\arctan \left(z^{\prime}(y)\right)\right) d y
$$

Finally, the total power of incident laser beam collected by entire aspheric lens can be performed in the following expression:

$$
P_{i}=\int_{-19 m m}^{0 m m} \frac{P_{a}}{\cos \arctan \left(z^{\prime}(y)\right)} \sin \left(\theta-\arctan \left(z^{\prime}(y)\right)\right) d y
$$

Taking into consideration of the incident angle $\frac{\pi}{2}-\gamma_{n}$, the total light power falling 
into the active area of photodiode can be express as

$$
P_{d}=\int_{y_{\max }}^{y_{\min }} \frac{P_{a}}{\cos \arctan \left(z^{\prime}(y)\right)} \sin \left(\theta-\arctan \left(z^{\prime}(y)\right)\right) \cos ^{2}\left(\frac{\pi}{2}-\gamma_{n}\right) d y
$$

$\left(y_{\min }, y_{\max }\right)$ is the effective incident region on the aspheric surface, which means incident rays only in this special range could be detected by photodiode and converted to effective electrical signal.

Taking $D=5 \mathrm{~m}$ as an example $\left(P_{a}=0.072 \mathrm{~mW} / \mathrm{mm}\right)$, the relationships between $P_{i}, P_{d}$ and incident angle $\theta$ are described in Fig. 8(a). In addition, on the basis of the average power received by the detector $P_{d}$, responsivity of photodiode $R$ $(0.64 \mathrm{~A} / \mathrm{W})$ and duty ratio of pulsed laser $T(0.1 \%)$, the theoretical peak photocurrent $I_{d}=P_{d} R / T$ can be calculated in different incident angle $\theta$. The comparison between calculated and experimental $I_{d}$ in various angle $\theta$ is shown in Fig. 8(b) while the experimental data $I_{d}$ originated from the amplified photocurrent $I$ (yellow solid diamond in Fig. 6). It is clear that the varying trend of experimental and calculated curves is almost same, although the value of experimental data is smaller than the calculated value at every tested angle. The primary factor causing the difference between two curves is that the laser scanning plane is not real ideal, which partially accords with the $2 \mathrm{D}$ geometrical model we developed. The distribution of laser 
plane in X-axis should also be considered, especially for circle-shaped photodiode rather than square-shaped photodiode, which would be improved in appropriate 3D geometrical model of OSPD in further research.

\section{CONCLUSION AND FUTURE PROSPECTS}

In conclusion, we have proposed and presented a miniaturized OSPD, being composed of a coated aspheric lens and a commercial single-point photodiode with small active area, that enables large-scale coordinate metrology. First of all, we derive the surface equation of the aspheric lens using vector theory in the geometry of $2 \mathrm{D}$ model and LightTools Software. For proof of principle, characterizing the OSPD with vertical $-5^{\circ}$ to $45^{\circ}$ and horizontal $360^{\circ}$ laser beam is experimentally demonstrated, which offers the unique capabilities to receive laser beam from multiple stations in entire space. Furthermore, the analytic and simulation results are also discussed, and it is indicated that a better OSPD could be constructed by further optimizing parameters when designed in developed 3D model.

The features of our device combined with spherical detector and omnidirectional detector using fisheye lens are particularly described in Table 1. Considering the fundamental structure of presented device, the accuracy of spatial locating depends on the size of photodiode instead of the apparent size of the detector when the relative 
position between photodiode and aspheric lens are precisely fixed. In addition, due to the reason that all scanning beams were collected by the small-sized photodiode, the detection speed of our device totally relies on the performance of photodiode, which could achieve $25 \mathrm{MHz}$ in spatial metrology. Compared to the conventional omnidirectional detectors, our device takes advantages of simple structure, fast response and precisely locating (shown in Table 2).

Table 1. Characteristics of three typical omnidirectional photodetectors

\begin{tabular}{|c|c|c|c|c|}
\hline & Speed & Sensitivity & Acceptance angle & Apparent size of detector \\
\hline Spherical detectors $^{7-10}$ & $\sim \mathrm{kHz}$ & $-36.45 \mathrm{dBm}$ & $360^{\circ}$ & Usually Large volume \\
\hline Fisheye lenses $^{11-13}$ & $\sim \mathrm{MHz}$ & $-50 \mathrm{dBm}$ & $90^{\circ}-180^{\circ}$ & Depending on the fisheye lens \\
\hline Our device & $25 \mathrm{MHz}$ & $-58 \mathrm{dBm}$ & $50^{\circ}$ & Small size \\
\hline
\end{tabular}

Table 2. Advantages and disadvantages of three typical omnidirectional photodetectors

\begin{tabular}{|c|c|c|}
\hline & Advantages & Disadvantages \\
\hline Spherical detectors ${ }^{7-10}$ & $360^{\circ}$ FOV both in horizontal and vertical & Low-speed, low-sensitivity \\
\hline Fisheye lenses ${ }^{11-13}$ & Large FOV, high-sensitivity & Complex, severe distortion \\
\hline Our device & Simple, high-speed, high-sensitivity & Limited FOV \\
\hline
\end{tabular}

What is more, our newly-developed design also provides the potential application to a diverse range, for instance, omnidirectional vision sensor, indoor global positioning system and optical wireless communication. Some challenges remain before the product of OSPD can be fully implemented, especially for further reducing the volume and largely degrading the effect of existed glass shell. In addition to using 
it independently, combining two identical OSPDs together would extend the detecting range to $-45^{\circ} \sim 45^{\circ}$ in vertical. We believe that the approach proposed in the article will inspire the future large-scale metrology, and facilitates a wide range of applications.

\section{ACKNOWLEDGMENTS}

We are grateful to Dr. Linghui Yang for useful discussion. This research was supported in part by the Aeronautical Science Foundation of China (No. 20165848005) and the Seed Foundation of Tianjin University (No. 2017XZS-0026).

\section{REFERENCES}

1. G. N. Peggs, P. G. Maropoulos, B. Hughes, and B. Muralikrishnan, "Recent developments in large-scale dimensional metrology," Proceedings of the Institution of Mechanical Engineers Part B Journal of Engineering Manufacture 223, pp. 571-595, 2009. [doi:10.1243/09544054JEM1284].

2. W. Li, X. Su, and Z. Liu, "Large-scale three-dimensional object measurement: a practical coordinate mapping and image data-patching method," Appl. Opt. 40, pp. 3326-3333, 2001. [doi:10.1364/AO.40.003326].

3. W. T. Estler, K. L. Edmundson, G. N. Peggs, and D. H. Parker, "Large-scale 
metrology an update," CIRP Annals - Manufacturing Technology 51, pp. 587609, 202. [doi:10.1016/S0007-8506(07)61702-8].

4. W. Cuypers, N. V. Gestel, A. Voet, J.-P. Kruth, J. Mingneau, and P. Bleys, "Optical measurement techniques for mobile and large-scale dimensional metrology," Optics and Lasers in Engineering 47, pp. 292-300, 2009. [doi:10.1016/j.optlaseng.2008.03.013].

5. T. Takatsuji, M. Goto, T. Kurosawa, Y. Tanimura, and Y. Koseki, "The first measurement of a three-dimensional coordinate by use of a laser tracking interferometer system based on trilateration," Measurement Science and Technology 9, pp. 38-41, 1998. [doi:10.1088/0957-0233/9/1/006].

6. Z. Huang, J. G. Zhu, L. H. Yang, J. W. B. Xue, and Z. Y. Zhao, "Accurate 3-d position and orientation method for indoor mobile robot navigation based on photoelectric scanning," IEEE transactions on instrumentation and measurement 64, pp. 2518-2529, 2015. [doi:10.1109/TIM.2015.2415031].

7. A. Kaandan, H. Refai, and P. Lopresti, "Wide-area and omnidirectional optical detector arrays using modular optical elements," Appl. Opt. 55, pp. 4791-4800, 2016. [doi:10.1364/AO.55.004791].

8. I. Giomataris, I. Irastorza., I. Savvidis, S. Andriamonje1, S. Aune1, M. Chapel- 
lier, P. Charvin, P. Colas, J. Derre1, E. Ferrer, M. Gros, X. F. Navick, P. Salin, and J. D. Vergados, "A novel large-volume spherical detector with proportional amplification read-out," Journal of Instrumentation 3, pp. 1531-1535, 2008. [doi:10.1088/1748-0221/3/09/P09007].

9. L. Gottardi, "Complete model of a spherical gravitational wave detector with capacitive transducers: Calibration and sensitivity optimization," Physical Review D 75, p. 022002, 2007. [doi:10.1103/PhysRevD.75.022002].

10. X. J. Huang, S. L. Chen, and S. X. Guo, "A 3d localization approach for subsea pipelines using a spherical detector," IEEE Sensors Journal 17, pp. 1828-1836, 2017. [doi:10.1109/JSEN.2016.2586998].

11. M. Drulea, I. Szakats, A. Vatavu, and S. Nedevschi, "Omnidirectional stereo vision using fisheye lenses," IEEE International Conference on Intelligent Computer Communication and Processing , pp. 251-258, 2014. [doi:10.1109/ICCP.2014.6937005].

12. S. Urban, J. Leitloff, and S. Hinz, "Improved wide-angle, fisheye and omnidirectional camera calibration," Isprs Journal of Photogrammetry and Remote Sensing 108, pp. 72-79, 2015. [doi:10.1016/j.isprsjprs.2015.06.005].

13. A. Mayra, M. Aikio, K. Ojala, S. Mandici, A. Vatavu, and S. Nedevschi, "Fish- 
eye optics for omnidirectional stereo camera," IEEE International Conference on Intelligent Computer Communication and Processing, pp. 225-230, 2015. [doi:10.1109/ICCP.2015.7312634].

14. C. Hughes, P. Denny, E. Jones, and M. Glavin, "Accuracy of fish-eye lens models," Appl. Opt. 49, pp. 3338-3347, 2010. [doi:10.1364/AO.49.003338].

15. T. Svoboda, T. Pajdla, and V. Hlaváč, "Epiplolar geometry for panoramic cameras," The fifth European Conference on Computer Vision, pp. 218-232, 1998. [doi:10.1007/BFb0055669].

16. T. Svoboda and T. Pajdla, "Epipolar geometry for central catadioptric cameras," International Journal of Computer Vision 49, pp. 23-37, 2002. [doi:10.1023/A:1019869530073]. 


\section{Biographies}

Hongbo Xie received the B.S., M.S. and Ph.D. degrees in Optical Engineering from Tianjin University, Tianjin, China, in 1992, 1994 and 1998, respectively. From 2003 to 2004, he worked in the Hong Kong University of Science and Technology, Hong Kong, China, as an academic visitor. He is an associate Professor with the Key Laboratory of Optoelectronics Information Technology (Ministry of Education), Tianjin University. His current research interests include optical system design and optical imaging.

Chensheng Mao received the B.S degree in Opto-electrical Engineering from Tianjin University, China, in 2015. He is currently a master candidate of Optical Engineering in Tianjin University. His research interests lie in optical design and infrared imaging.

Yongjie Ren is currently an associate professor with the State Key Laboratory of Precision Measurement Technology and Instruments, Tianjin University, Tianjin, China. He engages in laser and photoelectric measurement technology research. He has undertaken numbers of military projects, 2 National Natural Science Foundation projects and 1 National Major Instruments project. He also won the second prize of National Technical Invention and the first prize of Tianjin Technical Invention. 
Jigui Zhu received the B.S. and M.S. degrees from the National University of Defense Science and Technology, Changsha, China, in 1991 and 1994, respectively, and the Ph.D. degree from Tianjin University, Tianjin, China, in 1997. He is currently a Professor with the State Key Laboratory of Precision Measurement Technology and Instruments, Tianjin University. His current research interests include laser and photoelectric measuring technology, such as industrial online measurement and large-scale precision metrology.

Chao Wang received the Ph.D. degree in Electrical and Computer Engineering from the University of Ottawa, Canada, in 2011. From 2011 to 2012, he was a NSERC Postdoctoral Fellow in the Photonics Lab, University of California, Los Angeles (UCLA), USA. He is currently a Senior Lecturer in the School of Engineering and Digital Arts, University of Kent, UK. His research interests lie in microwave photonics, biophotonics, and optical communications.

Lei Yang received the B.S., M.S. and Ph.D. degrees in Optical Engineering from Tianjin University, Tianjin, China, in 2004, 2006 and 2009, respectively. He worked in School of Engineering and Digital Arts, University of Kent, UK, as an academic visitor in 2017. He is an assistant Professor with the Key Laboratory of Optoelectronics Information Technology (Ministry of Education), Tianjin University. His 
current research interests include optical design, ultrafast optical imaging and quantum optics. 


\section{Caption list}

Fig. 1 Principle of large-scale spatial coordinate measurement based on optoelectronic scanning. TS, transmitter station.

Fig. 2 Geometric model of OSPD, composed of a convex lens and a photodiode, in the coordinate system. Point $\mathrm{O}, \mathrm{V}, \mathrm{F}$ denote the origin, vertex and focus point, respectively. $\mathbf{p}, \mathbf{q}, \mathbf{n}$ are the unit vectors of incident ray, reflected ray and normal of the surface.

Fig. 3 Model of OSPD established in LightTools Software and its simulation results. (a) The ray-tracing model of OSPD when the angle of incident light $\theta$ ranges from $-5^{\circ}$ to $45^{\circ}$. The incident angle $\theta$ difference between adjacent light ray is $5^{\circ}$. (b) Spot pattern of light incoming the photodiode. The graphs (I) to (XI) originates from light with $-5^{\circ}$ to $45^{\circ}$ incident angle, when the difference of $\theta$ between two adjacent graphs is $5^{\circ}$. The legend representing the irradiation illumination of incident light with unit of $\mathrm{W} / \mathrm{mm}^{2}$.

Fig. 4 A photograph of assembled OSPD marked by outline dimension, consisting of a coated optical aspheric lens and a high-performance photodiode (First Sensor, PC5-6-TO5). The diameter of active area on photodiode is $2.52 \mathrm{~mm}$. 
Fig. 5 (a) Block diagram for testing the performance of OSPD. The signals outputting from OSPD are captured by oscilloscope (Tektronix, TDS2012B), when the relative angle $\theta$ between TS and OSPD (incident angle of laser beam) is varied by changing the relative working distance $D$ and height $H$. (b) A photograph of experimental setup in the lab. TS, transmitter station; Amp, amplifier; OSCP, Oscilloscope.

Fig. 6 The peak photocurrent $I$ outputting from OSPD versus the vertical incident angle $\theta$ of transmitter station when the relative working distance $D$ varies. Two-stage amplification circuit is employed for amplifying photocurrent by $20 \mathrm{~dB}$ before recording. The blue solid circle, red solid square, orange solid diamond, green solid triangle, purple hollow circle, blue hollow square, red hollow triangle represent the $D$ are $1 \mathrm{~m}, 3 \mathrm{~m}, 5 \mathrm{~m}, 7 \mathrm{~m}, 10 \mathrm{~m}, 11 \mathrm{~m}$ and $13 \mathrm{~m}$, respectively. The inset shows the measured average power of laser plane in unit interval $P_{a}$ when the distance $D$ between laser power meter (Ophir, Nova II) and transmitter station varies.

Fig. 7 Detailed analysis on the experimental arrangement. (a) Geometrical model of OSPD with parallel rays incoming. (b) The density map of $\gamma_{n}$ versus the incident angle $\theta$ and coordinate $y$. (c) The distribution of light spot on the $Z=0$ versus the incident angle of $\operatorname{OSPD} \theta$. The red bar denotes active area of 
photodiode on $Z=0$, which is from $-1.26 \mathrm{~mm}$ to $1.26 \mathrm{~mm}$. The red hollow square and circle stand for the maximum and minimum coordinate of light spot on $Z=0$, respectively. (d) The effective area of incident light on the aspheric surface, which accurately corresponds to the light spot in the active are of photodiode. Different color lines denote different incident angles, which are shown in the legend in detail.

Fig. 8 The numerical simulation results when the distance $D$ is fixed at $5 \mathrm{~m}$. (a) The average power of laser beam versus the incident angle $\theta$. The blue solid circle represents the total power of incident light collected by the entire aspheric surface $P_{i}$, corresponding to the primary axis. The red hollow square represents the power of reflected ray collected by photodiode $P_{d}$, corresponding to the secondary axis. (b) Photocurrent $I_{d}$ outputting from photodiode versus the incident angle $\theta$. The red hollow triangle represents the calculated photocurrent using the light power $P_{i}$ and experimental parameters. The yellow hollow diamond represents the obtained experimental data from Fig. 6 (yellow solid diamond) and relationship $I_{d}=10^{-2} I$. 